\title{
Efficacy of cognitive behavioral therapy in reducing psychiatric symptoms in patients with implantable cardioverter defibrillator: an integrative review
}

\author{
A.C.C.O. Maia ${ }^{1,2}$, A.A. Braga ${ }^{1,2}$, G. Soares-Filho ${ }^{1,2}$, V. Pereira ${ }^{1,2}$, A.E. Nardi ${ }^{1,2}$ and A.C. Silva ${ }^{1,2}$ \\ ${ }^{1}$ Laboratório de Pânico e Respiração, Instituto de Psiquiatria, Universidade Federal do Rio de Janeiro, Rio de Janeiro, RJ, Brasil \\ ${ }^{2}$ Programa de Pós-Graduação em Psiquiatria e Saúde Mental, \\ Instituto Nacional de Ciência e Tecnologia em Medicina Translacional, Rio de Janeiro, RJ, Brasil
}

\begin{abstract}
This article is a systematic review of the available literature on the benefits that cognitive behavioral therapy (CBT) offers patients with implanted cardioverter defibrillators (ICDs) and confirms its effectiveness. After receiving the device, some patients fear that it will malfunction, or they remain in a constant state of tension due to sudden electrical discharges and develop symptoms of anxiety and depression. A search with the key words "anxiety", "depression", "implantable cardioverter", "cognitive behavioral therapy" and "psychotherapy" was carried out. The search was conducted in early January 2013. Sources for the search were ISI Web of Knowledge, PubMed, and PsycINFO. A total of 224 articles were retrieved: 155 from PubMed, 69 from ISI Web of Knowledge. Of these, 16 were written in a foreign language and 47 were duplicates, leaving 161 references for analysis of the abstracts. A total of 19 articles were eliminated after analysis of the abstracts, 13 were eliminated after full-text reading, and 11 articles were selected for the review. The collection of articles for literature review covered studies conducted over a period of 13 years (1998-2011), and, according to methodological design, there were 1 cross-sectional study, 1 prospective observational study, 2 clinical trials, 4 case-control studies, and 3 case studies. The criterion used for selection of the 11 articles was the effectiveness of the intervention of CBT to decrease anxiety and depression in patients with ICD, expressed as a ratio. The research indicated that CBT has been effective in the treatment of ICD patients with depressive and anxiety symptoms. Research also showed that young women represented a risk group, for which further study is needed. Because the number of references on this theme was small, further studies should be carried out.
\end{abstract}

Key words: Anxiety; Depression; Cognitive behavioral therapy; Psychotherapy; Cardiology; Implantable cardioverter defibrillators

\section{Introduction}

The implantable cardioverter defibrillator (ICD) is a lifesaving device inserted into the heart of cardiac subjects that detects and attempts to correct potentially lethal ventricular arrhythmias with electrical discharges that stimulate the heart. Its use has become common among patients and, as a result, the mortality rate has been reduced from 54 to $30 \%$ (1). However, studies show that its use is related to a decline in the quality of life for patients, especially in the psychosocial area (1), and from 30 to $60 \%$ of ICD patients have various psychological complications, such as anxiety disorders and depressive episodes (2).
For some patients, shock is an extremely unpleasant event, both physically and emotionally (3), and the sensation caused by the electrical discharge from the device can be compared to a "kick in the chest", and, on a pain scale from 0 to 10 , this shock gets a score of 6 (4). Due to the tension and fear of receiving a sudden shock, as well as fear of the device not working properly or fear that any activity of daily life may cause an electrical discharge, people with ICDs are vulnerable to the onset of symptoms linked to anxiety disorders and depressive episodes (5).

Correspondence: A.C.C.O. Maia, Laboratório de Pânico e Respiração, Instituto de Psiquiatria, Universidade Federal do Rio de Janeiro, R. Visconde de Pirajá, 407/702, 22410-003 Rio de Janeiro, RJ, Brasil. Fax: +55-21-2523-6839. E-mail: acornelas@yahoo.com.br 
The most common concern of patients with the device is that it will not work properly, resulting in death, and that physical activities can alter the functioning of the device (1). As a result of fear and anxiety caused by catastrophic thoughts, the patient assumes an avoidance behavior for leisure activities, exercise, and sex (6).

Studies show that 24 to $37 \%$ of patients with an ICD have shown an increase in anxiety symptoms, and 13 to $35 \%$ are clinically diagnosed with anxiety disorder. Depression affects 13 to $21 \%$ of the patients (7). Studies indicate that cognitive behavioral therapy (CBT) is an effective tool in managing stress and symptoms related to anxiety, as well as for the minimization of catastrophic thoughts related to depressive symptoms in patients with ICDs (5-10).

CBT is a strategy or treatment approach proposing that emotional and behavioral problems arise as a result of a distorted or dysfunctional way of perceiving events, and influences friendliness and behavior. When an individual has his behavior and feelings determined by how he observes the world through his thoughts and beliefs, these are related to the world, the future, and himself. CBT aims to promote a cognitive restructuring through interventions in search of the emotional wellbeing of the individual (5-8). To this end, it is necessary to identify the beliefs, automatic thoughts, and cognitions of the individual (8).

The goal of psychotherapy for these patients is to improve their quality of life through a survey of skills, with the aim of increasing confidence before using the device as well as promoting psychosocial gains (9). Techniques using diaphragmatic breathing and progressive muscle relaxation exercises are employed in the management and administration of stress and anxiety treatments (5).

The aim of this study was to identify the benefits that CBT can offer patients with ICDs, through a systematic review of the clinical trials that used CBT to treat such patients.

\section{Material and Methods}

This study was conducted at the Laboratory of Panic and Respiration, Institute of Psychiatry, Federal University of Rio de Janeiro, and the National Institute of Science and Technology in Translational Medicine.

The terms used in the searches of scientific databases such as the ISI Web of Knowledge and PubMed were "anxiety", "depression", "implantable cardioverter", "cognitive behavioral therapy" and "psychotherapy".

An integrative review was chosen for this study because it allows inclusion of empirical and theoretical literature as well as studies with different methodological approaches, both quantitative and qualitative $(11,12)$. The studies included in this review were analyzed in a systematic manner in relation to aims and materials and methods. The intent was to provide the reader with an analysis of existing knowledge about treatments involving CBT and a discussion of the prevalent psychiatric problems that emerge after surgery for ICD. The integrative or systematic review, unlike traditional reviews, follows a set protocol that guides the entire review process from the identification of the problem, through the search for information, to the final report. The method used to select the studies in this review is presented, theories and evidence are discussed, relevant concepts are defined, and methodologies on a particular topic are analyzed in an attempt to find associations among elements in individual available studies $(11,12)$.

The survey was conducted in early January 2013; there was no restriction related to the year of publication of the articles from the databases. Incomplete articles and those not in the English language were excluded. Inclusion criteria were that only clinical trials and epidemiological studies were selected for this review. Inclusion criteria for clinical trials required the use of CBT as treatment for patients in the ICD population.

\section{Results}

A total of 224 references were found, 155 in PubMed and 69 in the ISI Web of Knowledge. A total of 63 were excluded because they were duplicates or because they were not in English. The abstracts of the 161 remaining references were analyzed and 13 were selected for fulltext analysis, because they dealt with the theme proposed in this review. Two were excluded because they were literature reviews. Eleven articles (Table 1) were included in the review because they matched the inclusion criteria: being a clinical trial, using CBT as treatment, and including a study population of ICD patients. The collection of selected articles comprised literature published over a period of 13 years (1998-2011). According to the methodological design as shown in Figure 1, there were 1 cross-sectional study, 1 prospective observational study, 2 clinical trials, 4 case-control studies, and 3 case studies.

\section{Prospective observational study}

The prospective observational study was conducted in the year 2000 and aimed to analyze the use of CBT to relieve distress following reduction of arrhythmias that required shock treatment. The sample consisted of 49 ICD patients, 25 who underwent psychotherapeutic intervention and a control group of 24 (13). CBT was used as a means of promoting psycho-education related to a decrease in the level of anxiety and constant fear of shocks, promoting better stress management, and resuming professional and social activities. The intervention was structured over 9 months. The average numbers of shocks received by patients in the two groups were not different (2.85 in the CBT group and 2.3 in the control group) (13). 
Table 1. Published studies about cognitive-behavioral therapy (CBT) for the reduction of psychiatric symptoms in patients with implanted cardioverter defibrillators (ICD).

\begin{tabular}{llcl}
\hline Date & Reference & Sample & Delimitation \\
\hline 1998 & Smith et al. (5) & 1 & Case study \\
2000 & Kohn et al. (13) & 49 & Prospective study \\
2009 & Lewin et al. (15) & 192 & Case-control study \\
& & & \\
2004 & Frizelle et al. (14) & 22 & Case-control study \\
& & & \\
2009 & Hirsh et al. (19) & 1 & Case study \\
& & & \\
2010 & Vazquez et al. (16) & 29 & Case-control study
\end{tabular}

2011 Irvine et al. (17) $193 \quad$ Case-control study

1999 Sears et al. (18) 2 Case study

2006 Chevalier et al. (20) $70 \quad$ Clinical trial study

2010 Irvine et al. (21) $193 \quad$ Clinical Trial Study

2005 Steinke (22) $28 \quad$ Cross-sectional study
Although the mentioned case presented a favorable prognosis for the decrease in symptoms related to anxiety, depression, panic disorder and agoraphobia, after the intervention in CBT there was still a therapeutic deficit to deal with this contingent of ICD.

The 25 patients who underwent CBT showed a reduction of psychological symptoms associated with the use of ICD. However, CBT is not associated with the reduction of electrical shock caused by the device.

Over 6 months, a reduction was observed in symptoms of depression in the intervention group compared to the control group. A similar pattern was observed with anxiety, and fewer limitations in physical activities. The Cardiac Rehabilitation program was effective and had a low cost.

The objective was to evaluate the effect of a pilot study program of the cardiac rehabilitation of 12 weeks in 22 ICD patients. After the intervention, patients showed physical and emotional improvements.

After psychotherapeutic intervention to the patient, better adaptation to ICD was shown, as well as a reduction of symptoms of depression and anxiety.

Psychotherapeutic interventions provided improvements in anxiety levels and acceptance of the device at an interval of one month. It was found that younger women represented a group at risk, and that they can benefit from psychotherapeutic intervention.

The objective was to evaluate 8 sessions of cognitive-behavioral intervention oriented to the adaptation of patients with ICD. The sample was composed of 193 patients and 96 underwent CBT and 97 formed the control group. It was concluded that CBT was an effective intervention in the adaptation of patients with ICD.

Both cases showed increased control of the thoughts that triggered anxiety. Classical conditioning and learned helplessness can be seen to explain the etiology of symptoms. It was demonstrated that cognitive-behavioral interventions provided a gain in the quality of life for patients.

Cognitive-behavioral therapy was administered to 70 patients with ICD. Although not presenting conclusive results, there was a decrease in anxiety levels.

The study concluded that women are more engaged with the therapeutic process. This study evaluated symptoms linked to depression, anxiety disorder, posttraumatic stress and quality of life. At the end of the intervention, all of these factors showed improvement except anxiety.

Essay on sexual counseling strategies and self-management with cardiac patients with ICD.
The results of this study indicated that CBT is an effective strategy for minimizing the psychological symptoms associated with the use of ICD. However, CBT was not associated with a reduction of electrical discharges (13).

\section{Case-control studies}

Four case-control studies were found. The first, published in 2004 was a pilot study that evaluated a 12-week pilot study of a cardiac rehabilitation program in ICD patients (14). The sample was composed of 22 ICD patients (14) randomized to either the cardiac rehabilitation program or a control group. All were assessed at baseline, at the end of the study, and 3 months after the intervention, to determine the effects of the program (14).

In the group that took part in the program, there were positive changes, although small. According to the Hospital Anxiety and Depression Scale (HADS), occurrence of symptoms improved, from 25 to $17 \%$ for those who underwent the intervention, and from 24 to $20 \%$ for the control group. Globally, the study found evidence that a cardiac rehabilitation program provided significant physiological and psychological benefits (14).

Another study in 2009 investigated the cost effectiveness of a therapy for heart patients, based on cognitive 


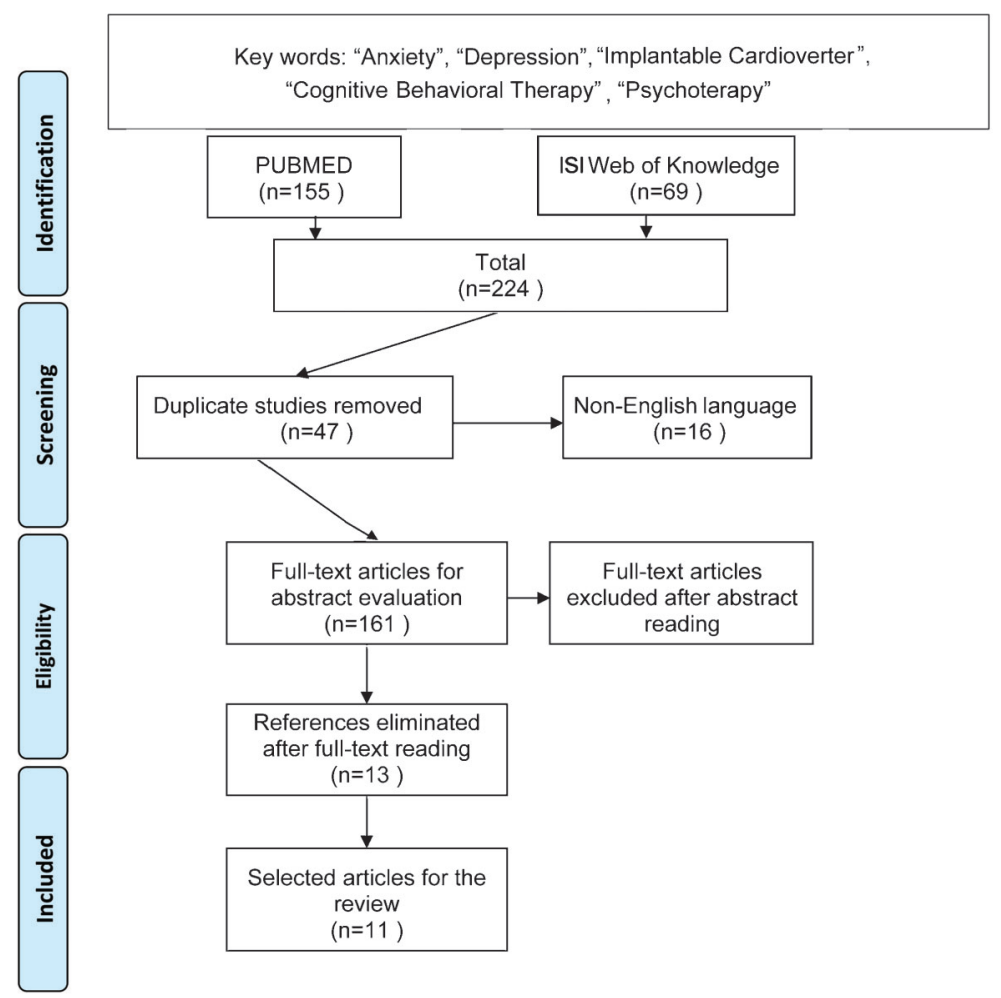

Figure 1. Results of the integrative review.

and behavioral components. Of 192 patients, 71 experienced a psychotherapeutic intervention consisting of a booklet for the family, a daily goal-setting diary, and a relaxation tape (15)

Over 6 months, the reduction in symptoms of depression in the intervention group was significantly greater than in the control group. A similar pattern was observed with anxiety, and fewer limitations in physical activities were observed. The intervention proved to be effective and lowcost (15)

A study in 2010 aimed to implement and verify the effectiveness of group therapy to promote the reduction of symptoms related to anxiety in women with ICD. The sample was composed of 29 women, 14 who underwent therapeutic intervention and 15 who were a control group (16).

The Florida Shock Anxiety Scale (FSAS) was used to measure anxiety levels linked to sudden shocks. The women given CBT had scores of 22.79 before the intervention and 17.73 at the end of the intervention. The control group had corresponding scores of 19.93 and 19.13 (16). The FSAS was also used to measure patient acceptance of the ICD unit. The control group scores showed no significant change, i.e., from 70.89 to 71.11 within 1 month. The intervention group scores at the beginning and end of the treatment were 69.52 and 78.93 , respectively. Psychotherapeutic intervention provided improvements in anxiety levels and acceptance of the device in an interval of 1 month. It was found that younger women represented a risk group, and there may be benefits from psychotherapeutic intervention (16).

In 2011, a survey was conducted to evaluate an intervention of eight sessions of CBT aimed at the adaptation of ICD patients. This sample consisted of 193 patients, 96 of whom underwent CBT and 97 were controls. The survey concluded that CBT was an effective intervention in the adaptation of patients to ICD (17).

\section{Reports of case studies}

Three case studies were conducted. The first was in 1998 and aimed to describe the psychological reaction and management of a clinical case (5). In addition to the stress associated with heart disease, the patients with the device faced constant fear of shock and dependency on the device. They represented a population vulnerable to anxiety and depression. Research shows that $50 \%$ of these patients are at risk of developing a psychiatric disorder, $30 \%$ a mixed disorder, $15 \%$ depressive episodes, and $5 \%$ panic (5).

The research focused on a patient diagnosed with dysplasia of the right ventricle. After receiving several electrical discharges, she began to show symptoms of hypervigilance, nightmares, and agoraphobia in addition to dizziness, fatigue, and nausea, which were not caused by use of an automatic ICD. As time went on, she experienced panic attacks, an intensification of agoraphobic symptoms and generalized anxiety disorder (5). 
Her symptoms remained unchanged over two sessions. She was also diagnosed using the Diagnostic and Statistical Manual Fourth Edition Chapter 10 (6), with the following comorbidities: panic disorder with agoraphobia, major depressive disorder, and post-traumatic stress disorder resulting from a rape she suffered 14 years earlier. Her therapy was conducted in eight weekly sessions, tapering to seven sessions over 10 months.

With the implementation of the Fear Questionnaire, an improvement was seen in symptoms associated with agoraphobia (from 16 to $5 \%$ ) and dysphoria (from 26 to $13 \%$ ), but the levels of social phobia remained unchanged ( $2 \%$ at the beginning and at the end of psychotherapeutic intervention). The Beck Depression Inventory (BDI) also showed an improvement in depressive symptoms (from 19 to $13 \%)(5)$.

Although the reported case presented a favorable prognosis, including decreases in symptoms related to anxiety, depression, panic disorder, and agoraphobia, a deficit still exists in the therapy to deal with this category of reactions to an automatic ICD (5).

The authors concluded that, facing the increasing complexity of medical interventions to prolong the lives of sick patients, the development and implementation of interventions based on CBT can provide gains in quality of life for the patients (5).

In 1999, a study following two patients with ventricular tachycardia sought to understand how psychopathological symptoms developed with the use of the ICD device (18). The ICD increased the life expectancy of the patients but, psychological complications such as levels of anxiety and constant fear of a shock increased (18).

A 51-year-old patient had three episodes of electrical discharges caused by the device in two hospitalizations for chest pain. Psychotherapy was begun 4 years after ICD implantation following a diagnosis of depressive disorder and anxiety. It was reported that the device drastically affected social life and quality of life. The second patient was 58 years old and was hospitalized twice after receiving more than 40 electrical discharges. The therapy started 2 weeks after implantation when diagnosed with mixed anxiety disorder and depressed mood. As a result, the patient showed an improvement in the context of anxiety, but not in depressive symptoms (18).

Both cases showed an increase in the ability to control the thoughts that triggered anxiety. Classical conditioning and learned helplessness can be observed to explain the etiology of symptoms. It was demonstrated that cognitive behavioral interventions provided gains in quality of life for the patients.

In 2009, a case study was reported of a 50-year-old patient with a history of ischemic cardiomyopathy, chronic atrial fibrillation, diabetes, dyslipidemia, morbid obesity, and gout (19). The patient was referred to a psychologist because of the presence of a constant affliction and dynamically significant symptoms of anxiety and depression. Fear of shocks led him to avoid leisure activities, exercise, and sex. Depressive symptoms were measured using the BDI, and an initial score of 18 fell to 0 after 6 weeks of psychotherapy. On the FSAS, which correlates and measures the level of anxiety with sudden shocks, the patient scored 30 before and 14 after psychotherapy. At the conclusion of the process, he showed better adaptation to the ICD as well as a reduction in symptoms of anxiety and depression (19).

\section{Clinical trials}

Two clinical trials were found during the search. The first was conducted in 2006, and its goal was to determine whether CBT decreased arrhythmic events and promoted the well-being of ICD patients (20). A sample of 253 ICD patients was selected, of which 70 were recruited to participate in the study. The patients were given CBT for 12 months during which psychological well-being, quality of life and automatic tone were evaluated. The BDI-13 was the instrument used to evaluate depressive symptoms. The CBT and control groups showed initial scores of 6.46 and 4.56, and after 12 months the results were 4.92 and 6.06 , respectively. Anxiogenic symptomatology also showed favorable scores through data collected using the Hamilton Anxiety Scale. The CBT and control groups scored 6.15 and 5.75 , respectively, in the first month and 2.62 and 9 by 12 months. The study did not provide conclusive results because of obstacles such as patients' refusal to complete the psychological tests, a high dropout rate, and the difficulty of convincing patients that problems with stress could be damaging to health (20).

The second clinical trial was conducted in 2010 with the objective of evaluating acceptance of CBT in cardiac patients as a facilitator of adaptation to an ICD and determining whether gender is associated with engagement in therapy (21). Ninety-six patients were selected for the intervention sample. This study indicated that women are more likely to develop psychological problems after ICD implantation (21). Patients were offered eight counseling sessions, a psycho-educational booklet, a CD-ROM with relaxation exercises, and five sessions by telephone, which were planned in case the patient had difficulty leaving home.

From 66 to 77 patients rated the advice and the booklet as "very to extremely useful". The survey revealed that $29.7 \%$ of patients participated in four or fewer sessions, $25.9 \%$ attended five to seven, and $44.4 \%$ attended eight or more. The results of the tools provided showed significant improvement in the patients except for the HADS anxiety results. The study confirmed that women showed a greater response to treatment than men did (21).

\section{Cross-sectional study}

Only one cross-sectional study was found. It was an 
assessment of strategies for sexual counseling and selfmanagement in ICD patients (22). It pointed out that more research is needed to determine the needs, concerns, and teaching strategies related to the sex lives of older people (22).

\section{Discussion}

There are several studies on psychological intervention for help in adapting to the ICD (23). Nevertheless, there are few studies that compare the efficacy of different approaches for the treatment of psychiatric symptoms in these patients. The available literature shows that ICDs keep patients alive through the detection and correction of potentially lethal ventricular arrhythmias by delivering electrical discharges that stimulate the heart. The mortality rate decreases to $3 \%$ per year among patients at high risk of heart failure after the implantation of an automatic ICD (22). However, as activation of the device is unpredictable and sometimes painful, the shock becomes an unpleasant event for the patient, causing avoidance of working situations, physical exercise, and sex, because the patient associates these activities with the occurrence of electrical discharges (24). They also fear that the device will stop functioning at any time or will operate improperly, and this leaves them in a constant state of tension and stress.

Due to these factors, the ICD population becomes vulnerable to a number of psychological complications such as anxiety disorders and depressive episodes $(22,24)$. One study demonstrated that two processes could explain the appearance of symptoms: classical conditioning and learned helplessness. Classical conditioning can be observed in these ICD patients when they experience a painful shock (unconditioned stimulus) that produces anxiety or fear (unconditioned response). Random stimuli such as a physical activity, when associated with electrical discharges generated by the device, can trigger anxiety in the patient before the expected shock, which may not happen (23). Learned helplessness suggests that experiencing psychological distress leads patients to perceive that they are unable to handle situations because they judge the situations as uncontrollable (25).

It is also possible that the patients may develop symptoms associated with agoraphobia and panic disorder (5). Because these patients have distorted thoughts, which do not correspond to reality, and demonstrate avoidance behaviors resulting in personal and physical injury, CBT is recommended for these patients (26). The goal of psychotherapy is to strengthen the quality of life through cognitive restructuring and analysis of evidence, to strengthen confidence toward the use of the device, and to promote psychosocial gain (9). CBT does not aim to decrease the frequency or intensity of the shocks, but to make the patient's life healthier and more realistic with an ICD (6).

CBT was used as a means of promoting psychoeducation of patients and favored lowering their levels of anxiety and depressive symptoms linked to the constant fear of receiving an electric shock, as well as promoting better management of stress and the patient's return to daily activities (6). This approach aims to help patients modify dysfunctional beliefs and behaviors that produce certain moods (10).

The aim of CBT is to a) help patients identify their distorted beliefs about the use of the device, b) recognize the negative automatic thoughts and seek alternative thoughts more consistent with reality, and c) seek evidence to support the negative thoughts and the thoughts grounded in reality (15).

The most used relaxation technique is diaphragmatic breathing. It promotes a state of deep relaxation and consists basically of sitting or lying down comfortably. The patient is taught to inhale air through the nose, filling the lungs (15) and retaining the air for $2 \mathrm{~s}$, then exhaling slowly through the mouth. The procedure is repeated until the patient is more relaxed (16). This technique is used for patients who have high levels of stress and anxiety and who also have difficulty relaxing. ICD patients who are in a constant state of tension due to fear can benefit from this technique (5).

Cognitive distortion consists of errors in perception and information processing. During this process, people tend to structure their thoughts in a negative and inflexible way, resulting in errors of interpretation related to personal performance and the judgment of external situations $(14,17)$.

Some ICD patients interpret the electrical discharges as a signal that the device is malfunctioning or that it can stop working at any time (5). Some people may associate social activities, physical exercise, and sex as factors that increase the frequency of the electric shocks and so eventually avoid those activities (24).

Cognitive restructuring for these patients helps them to think more coherently and focus on reality. A cardiologist can be called upon to solve questions regarding the use and operation of the device $(5,9)$.

Other techniques can be employed in the treatment of ICD patients, such as a) establishing weekly goals to enable patients to return to pleasant activities of daily life; b) forming groups to facilitate the sharing of coping strategies, feelings, experiences, and the provision of emotional support from people in similar circumstances; and c) structuring a set of daily exercises compatible with the physical condition of the patient (5).

All selected studies showed that CBT constitutes an effective intervention in the reduction of psychopathological symptoms related to the use of the device. However, some studies $(5,9,25)$ concluded that CBT can deliver short-term results and that more research needs to be undertaken to refine the data collected so far. The authors emphasized that there are few findings that correlate the use of the device to psychopathological symptoms (1828). Some studies $(19,27)$ showed that young women 
represent a risk population regarding the onset of symptoms linked to depression and anxiety due to hormones related to pregnancy and the menstrual period.

\section{Conclusion}

CBT is not only safe but also promotes a better physical condition and provides lower stress levels in patients. CBT applied in this population promoted cognitive restructuring by replacing distorted thoughts and catastrophic thoughts through the use of evidence of reality. Doubts and misunderstandings related to the use of the device were clarified, expanding the scope of understanding by the patient about his or her medical

\section{References}

1. Dunbar SB. Psychosocial issues of patients with implantable cardioverter defibrillators. Am J Crit Care 2005; 14: 294-303.

2. Hegel MT, Griegel LE, Black C, Goulden L, Ozahowski T. Anxiety and depression in patients receiving implanted cardioverter-defibrillators: a longitudinal investigation. Int $J$ Psychiatry Med 1997; 27: 57-69, doi: 10.2190/1G9VEQMD-MTLQ-E0BW.

3. Burgess ES, Quigley JF, Moran G, Sutton FJ, Goodman M. Predictors of psychosocial adjustment in patients with implantable cardioverter defibrillators. Pacing Clin Electrophysiol 1997; 20: 1790-1795, doi: 10.1111/j.15408159.1997.tb03568.x.

4. Ahmad $M$, Bloomstein $L$, Roelke $M$, Bernstein $A D$, Parsonnet V. Patients' attitudes toward implanted defibrillator shocks. Pacing Clin Electrophysiol 2000; 23: 934-938, doi: 10.1111/j.1540-8159.2000.tb00877.x.

5. Smith LC, Fogel D, Friedman S. Cognitive-behavioral treatment of panic disorder with agoraphobia triggered by AICD implant activity. Psychosomatics 1998; 39: 474-477, doi: 10.1016/S0033-3182(98)71311-8.

6. American Psychiatric Association. The diagnostic and statistical manual of mental disorders. 4th edn. Washington: American Psychiatric Association; 1994.

7. Pilote L, Dasgupta K, Guru V, Humphries KH, McGrath J, Norris $\mathrm{C}$, et al. A comprehensive view of sex-specific issues related to cardiovascular disease. CMAJ 2007; 176: S1S44, doi: $10.1503 / \mathrm{cmaj} .051455$.

8. Seligman MEP. Helplessness: on depression, development, and death. San Francisco: WH Freeman and Company; 1999.

9. Powell VB, Abreu N, Oliveira IR, Sudak D. [Cognitivebehavioral therapy for depression]. Rev Bras Psiquiatr 2008; 30 (Suppl 2): S73-S80, doi: 10.1590/S151644462008000600004.

10. Sneed NV, Finch N. Experiences of patients and significant others with automatic implantable cardioverter defibrillators after discharge from the hospital. Prog Cardiovasc Nurs 1992; 7: 20-24

11. Whittemore R, Knafl K. The integrative review: updated methodology. J Adv Nurs 2005; 52: 546-553, doi: 10.1111/ j.1365-2648.2005.03621.x. condition. CBT is a form of effective intervention; however, further studies need to be carried out, since the number of references found on the subject is small. Young women, because they represent a risk group, can benefit from cognitive behavioral interventions.

This review was limited by not following a systematic review or meta-analysis model. That would have been difficult because "gold-standard" level studies are rare in the published literature on CBT treatment in patients with ICDs.

\section{Acknowledgments}

Research supported by CNPq.
12. Mata FGD, Neves FS, Lage GM, Moraes PHP, Mattos P Fuentes D, et al. Neuropsychological assessment of the decision making process in children and adolescents: an integrative review of the literature. Rev Psiq Clín 2011; 38 106-115, doi: 10.1590/S0101-60832011000300005.

13. Kohn CS, Petrucci RJ, Baessler C, Soto DM, Movsowitz C. The effect of psychological intervention on patients' longterm adjustment to the ICD: a prospective study. Pacing Clin Electrophysiol 2000; 23: 450-456, doi: 10.1111/j.1540-8159. 2000.tb00826.x.

14. Frizelle DJ, Lewin RJ, Kaye G, Hargreaves C, Hasney K, Beaumont $\mathrm{N}$, et al. Cognitive-behavioural rehabilitation programme for patients with an implanted cardioverter defibrillator: a pilot study. $\mathrm{Br} J$ Health Psychol 2004; 9: 381-392, doi: 10.1348/1359107041557039.

15. Lewin RJ, Coulton S, Frizelle DJ, Kaye G, Cox H. A brief cognitive behavioural preimplantation and rehabilitation programme for patients receiving an implantable cardioverterdefibrillator improves physical health and reduces psychological morbidity and unplanned readmissions. Heart 2009; 95: 63-69, doi: 10.1136/hrt.2007.129890

16. Vazquez LD, Conti JB, Sears SF. Female-specific education, management, and lifestyle enhancement for implantable cardioverter defibrillator patients: the FEMALE-ICD study. Pacing Clin Electrophysiol 2010; 33: 1131-1140, doi: 10.1111/j.1540-8159.2010.02787.x.

17. Irvine J, Firestone J, Ong L, Cribbie R, Dorian P, Harris L, et al. A randomized controlled trial of cognitive behavior therapy tailored to psychological adaptation to an implantable cardioverter defibrillator. Psychosom Med 2011; 73 226-233, doi: 10.1097/PSY.0b013e31820afc63.

18. Sears SF Jr, Conti JB, Curtis AB, Saia TL, Foote R, Wen F. Affective distress and implantable cardioverter defibrillators: cases for psychological and behavioral interventions. Pacing Clin Electrophysiol 1999; 22: 1831-1834, doi: 10.1111/j.1540-8159.1999.tb00422.x.

19. Hirsh AT, Sears SF Jr, Conti JB. Cognitive and behavioral treatments for anxiety and depression in a patient with an implantable cardioverter defibrillator (ICD): a case report and clinical discussion. J Clin Psychol Med Settings 2009; 16: $270-279$, doi: 10.1007/s10880-009-9160-0

20. Chevalier P, Cottraux J, Mollard E, Sai N, Brun S, Burri H, 
et al. Prevention of implantable defibrillator shocks by cognitive behavioral therapy: a pilot trial. Am Heart J 2006; 151: 191, doi: 10.1016/j.ahj.2005.10.007.

21. Irvine J, Stanley J, Ong L, Cribbie R, Ritvo P, Katz J, et al. Acceptability of a cognitive behavior therapy intervention to implantable cardioverter defibrillator recipients. J Cogn Psychother 2010; 24: 246-264, doi: 10.1891/0889-8391. 24.4.246.

22. Steinke EE. Intimacy needs and chronic illness: strategies for sexual counseling and self-management. J Gerontol Nurs 2005; 31: 40-50.

23. Beck JS. Cognitive therapy: basics and beyond. New York: Guildford Press; 1995.

24. Sears SF Jr, Todaro JF, Lewis TS, Sotile W, Conti JB. Examining the psychosocial impact of implantable cardioverter defibrillators: a literature review. Clin Cardiol 1999; 22: 481-489, doi: 10.1002/clc.4960220709.
25. Range BP, Marlatt GA. [Cognitive-behavioral therapy for alcohol and drug use disorders]. Rev Bras Psiquiatr 2008; 30 (Suppl 2): S88-S95, doi: 10.1590/S1516-4446200800 0600006.

26. Pelletier LG, Tuson KM, Haddad NK. Client Motivation for Therapy Scale: a measure of intrinsic motivation, extrinsic motivation, and amotivation for therapy. J Pers Assess 1997; 68: 414-435, doi: 10.1207/s15327752jpa6802_11.

27. Scher CD, Segal ZV, Ingram RE. Beck's theory of depression: origens, empirical status, and future directions for cognitive vulnerability. In: Leahy RL (Editor), Contemporary cognitive therapy: theory, research and practice. New York: Guilford Press; 2006.

28. Morris PL, Badger J, Chmielewsky C, Goldberg RJ. Psychiatric morbidity following implantation of the automatic implantable cardioverter defibrillator. Psychosomatics 2009; 32: 58-64, doi: 10.1016/S0033-3182(91)72112-9. 